\title{
Clinical and Radiological Outcomes in Arthroscopic Repair of Shoulder Rotator Interval Lesions
}

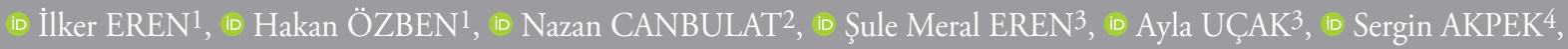 \\ iD Mehmet DEMIRHAN1 \\ ${ }^{1}$ Koç University Faculty of Medicine, Department of Orthopedics and Traumatology, İstanbul, Turkey \\ ${ }^{2}$ Koç University Faculty o Medicine, Department of Physical Therapy and Rehabilitation, İstanbul, Turkey \\ ${ }^{3}$ Vehbi Koç Foundation American Hospital, Clinic of Physical Therapy and Rehabilitation, İstanbul, Turkey \\ «Vehbi Koç Foundation American Hospital, Clinic of Radiological, İstanbul, Turkey
}

\begin{abstract}
Objective: Rotator interval lesion (RIL) is a distinct rotator cuff (RC) injury patern consisting of subscapularis and supraspinatus tear with biceps problem. This pathology is an underdiagnosed RC entitiy and not studied in-depth. Aim of this study is to report the functional and radiological results of RIL surgeries performed.
\end{abstract}

Methods: Surgeries performed in a single center, in a 7-year-period were retrospectively reviewed. Sixteen cases ( $\mathrm{n}=16)$ who underwent arthroscopic RC repair including subscapularis with biceps tenodesis or tenotomy were called for an up-to-date assessment. Fourteen shoulders of 13 patients (3 females, 10 males) were included. Constant, disabilities of the arm shoulder and hand (DASH), standardized shoulder assessment form (ASES) scores, and RC thickness measurements with magnetic resonance imaging (MRI) were recorded. Preoperative and postoperative results were compared.

Results: Average age of the patients were 60.6 (47-74), and follow-up period was 3.2 \pm 1.9 years. Average preoperative Constant, DASH and ASES scores were $44.43 \pm 15.4,22.11 \pm 17.21$ and $51.37 \pm 27.6$, respectively. Postoperative values improved to $90.45 \pm 6.44,6 \pm 13.68$ and $95.82 \pm 7.82$, respectively $(\mathrm{p}<0.05)$. Average subscapularis and supraspinatus thicknesses measured with MRI were $3.85 \pm 0.87$ and $4.60 \pm 0.65 \mathrm{~mm}$ respectively. MRI revealed subscapularis tendinitis in 1 patient. Re-tear was not observed in any patients.

Conclusion: Arthroscopic subscapularis and supraspinatus repair with biceps tenodesis or tenotomy is an effective treatment method in RIL. No retear was observed with MRI. Clinical results are similar with other RC pathologies.

Keywords: Rotator cuff, rotator interval, subscapularis, magnetic resonance imaging

\section{Introduction}

Rotator interval (RI) lesions (anterior-superior tears) represent a special subset of rotator cuff (RC) tears. In these lesions, pathology is localised to anterior structures, and they always present with supraspinatus tear, biceps tendon pathology and subcapularis tear (1). They were classified as type A tears by Collins et al. (2). RI lesions are thought to account for $4 \%$ of all RC tears.
A small number of articles have been published in the literature, due to the rare occurrence of them compared with posteriorsuperior tears. It is suggested that natural course and prognosis are worse than conventional supraspinatus and infraspinatus tears or isolated subcapularis tears (1). Good results are reported in arthroscopic treatment of subcapular tears (3), for this reason, it is thought that RI lesions with similar patho-anatomy will have good results with arthroscopic treatment $(3,4)$. However, due to
Address for Correspondence: Ilker EREN, Koç University Faculty of Medicine, Department of Orthopedics and Traumatology, istanbul, Turkey

Phone: +90 8502508250 E-mail: ilker.eren@gmail.com ORCID ID: orcid.org/0000-0003-2965-7690

Cite this article as: Eren I, Özben H, Canbulat N, Eren ŞM, Uçak A, Akpek S, Demirhan M. Clinical and Radiological Outcomes in Arthroscopic Repair of Shoulder Rotator Interval Lesions. Bezmialem Science 2019;7(3):204-7.
Received: 10.07 .2018

Accepted: 22.10.2018 
the small number of studies on the subject, the results are still considered to be unclear.

The hypothesis of this study is that the arthroscopic treatment of RI lesions is comparable to the arthroscopic treatment of other RC lesions reported in the literature and can be accepted as successful. For this purpose, functional clinical and magnetic resonance imaging (MRI) results in our patients who were diagnosed as having RI lesions and underwent arthroscopic repair were reported in this study.

\section{Methods}

Following the approval of the ethics committee, all RC tears operated by a single surgeon in a single center in 7-year period (2007-2014) were retrospectively screened. The inclusion criteria were: (1) Superior $1 / 3$ complete tendon tear of subcapularis tendon and repair, (2) Tenodesis or tenotomy due to biceps tendon pathology, (3) Supraspinatus tear and repair, (4) One year and over follow-up period and (5) Arthroscopic surgery used as surgical technique. Owners of sixteen shoulders which met the inclusion criteria among $337 \mathrm{RC}$ surgeries during the identified period were invited for functional evaluation and radiological examinations. Fourteen shoulder joints of 13 patients (10 males, 3 females) who agreed to participate in the study and completed the appropriate clinical and radiological examinations were included in the study.

All patients underwent the same surgical procedure. Following arthroscopic joint examination and biceps tenotomy with radiofrequency, subacromial decompression and acromioplasty were performed. Tendon footprints in large and small tubercles were prepared and tendon debridement was performed until healthy edge was obtained. For subscapularis repair, first suture, no: 2 high strength braided suture, is put from superior edge as a cement, and second suture is put from the torn body of the tendon in matrix form (Fiberwire, Arthrex, Florida, USA), and then, both of them are fixed on tendon footprints with the help of a nodeless anchor. Single medial anchor was used in supraspinatus and infraspinatus tears below $1.5 \mathrm{~cm}$, double medial anchors were used in tears above $1.5 \mathrm{~cm}$ and double lateral anchors were used in total tears. As a result, all of them were repaired with double raw method which is equivalent of nodeless transosseus method. In all patients, biocomposite absorbable anchor was used (Biocomposite Swivelock, Arthrex, Florida, USA). After the operation, the patients were followed up with a padded arm hanger for 1 month and activities on table were allowed immediately. Active assistive exercises were initiated at the end of the first month and resistive exercises were initiated at the end of the second month.

The patients were examined by a single physical therapist with over 15 years of shoulder and elbow surgery experience. Examination of subscapularis was performed using lift-off, bear-hug and belly-press tests (5-7) and clinical evaluation was performed using Constant, disabilities of the arm shoulder and hand (DASH) and standardized shoulder assessment form (ASES) scores (8-10). Preoperative Constant, ASES and DASH scores were obtained from the files of the patients.

MRI was performed in supine position using standard shoulder protocols (3T System; Skyra; Siemens Medical Solutions, Erlangen, Germany) and with a dedicated shoulder coil. T1weighted spin-echo images (TR/TE 420/11) were obtained in the sagittal oblique plan (parallel to glenohumeral joint) and proton density-T2 weighted images (TR/TE 2300-2800/4546) were obtained in the axial, sagittal and coronal oblique plan (straight to glenohumeral joint). The cross-sectional range was set to $3 \mathrm{~mm}$, inter-sectional range was set to $0.4 \mathrm{~mm}$ and the matrix dimensions were determined as $384 \times 288$. The field of study was between $14-18 \mathrm{~cm}$. The images were interpreted by a single radiologist with over 20 years of musculoskeletal radiology experience. Tendon thicknesses of supraspinatus, infraspinatus and subscapularis muscles were recorded as means of $\mathrm{mm}$. S.B. Koç University Faculty of Medicine Clinical Research Ethics Committee decision no: 2014.110.IRB1.001.

\section{Statistical Analysis}

Statistical analysis was performed using MedCalc 12.5 Statistical Software (Ostend, Belgium). In addition to descriptive statistical methods (mean and standard deviation), a dependent student's t-test was used to compare the clinical scores before and after surgery. P value below 0.05 was accepted as statistically significant.

\section{Results}

The mean age of 13 patients ( 10 males, 3 females) was 60.6 (47-74) years and the mean follow-up period was $3.2 \pm 1.9$ years. Tenodesis was performed in 9 shoulders and tenotomy in 5 shoulders for the pathology of biceps tendon.

Preoperative constant, DASH and ASES scores were found $44.43 \pm 15.4,22.11 \pm 17.21$ and $51.37 \pm 27.6$, respectively. These values were measured as $90.45 \pm 6.44,6 \pm 13.68$ and $95.82 \pm 7.82$ in the last control after the operation. The increase in all scores was statistically significant $(\mathrm{p}<0.05)$. Subcapularis tests (lift-off, belly-press, and bear-hug) were positive in only one shoulder, while they were negative in the other shoulders $(n=1 / 14)$.

Constant, DASH and ASES scores of the pathological shoulders of the patients are summarized in Table 1 .

Tendon thicknesses in MRI performed in the last control are summarized in Table 2. No re-tear was detected in any patient. Tendinitis of supraspinatus and subcapularis tendons were observed in a patient.

\begin{tabular}{|c|c|c|c|}
\hline & Constant & DASH & ASES \\
\hline Ргеор & $\begin{array}{l}44.43 \pm 15.39 \\
25-62\end{array}$ & $\begin{array}{l}22.11 \pm 17.21 \\
0-50\end{array}$ & $\begin{array}{l}51.37 \pm 27.60 \\
10-86.6\end{array}$ \\
\hline Postop & $\begin{array}{l}90.45 \pm 6.44 \\
79.46-98\end{array}$ & $\begin{array}{l}5.99 \pm 13.68 \\
0-49.13\end{array}$ & $\begin{array}{l}95.82 \pm 7.82 \\
73.3-100\end{array}$ \\
\hline$p$ & $<0.001 *$ & $0.001^{*}$ & 0.006 * \\
\hline
\end{tabular}


Table 2. Tendon thickness measurements obtained by magnetic resonance imaging

\begin{tabular}{|c|c|c|c|}
\hline & $\begin{array}{l}\text { Subscapularis } \\
(\mathrm{mm})\end{array}$ & $\begin{array}{l}\text { Supraspinatus } \\
(\mathrm{mm})\end{array}$ & $\begin{array}{l}\text { Infraspinatus } \\
(\mathrm{mm})\end{array}$ \\
\hline \multicolumn{4}{|c|}{ Magnetic resonance } \\
\hline Mean & $3.85 \pm 0.87$ & $4.60 \pm 0.65$ & $3.90 \pm 1.14$ \\
\hline Min-max & $2.80-5.3$ & $3.60-5.9$ & $1.40-5.7$ \\
\hline
\end{tabular}

\section{Discussion}

RI refers to the region between subscapularis and supraspinatus muscles, and includes biceps tendon, coracohumeral ligament and superior glenohumeral ligament. This region also includes the suspension system where the biceps tendon leaves the glenohumeral joint (11). Because of involving multiple structures, pathologies of this region are with involvement of both subscapularis and supraspinastus tendons, with biceps tendon pathologies, and affects the stability of the glenohumeral joint in superior-inferior direction. For this reason, it is considered as a separate entity and it has clinical characteristics.

Subcapularis tears was previously classified according to the tear width by Lafosse et al. $(12,13)$ And this classification is frequently used in clinical practice. Clinical results of subcapularis lesions with a spectrum ranging from partial superior $1 / 3$ tears (Lafosse I) to complete layer tears (Lafosse V) in which centralization of the head deteriorates, also vary according to the type of tear. There is still no consensus on how much the definition of "RI tear" covers subscapularis muscle. In order to obtain a homogenous group, only tears of Lafose type II (superior $1 / 3$ complete layer) were included in this study. Another publication that classifies the pathology of structures within RI belongs to Bennett (14). This classification is based on the relationship between subscapularis tear and coracohumeral and glenohumeral ligaments, the displacement of the biseps pulley and whether the subscapularis tear is intraarticular or complete layer. This classification was not used in our study, because patients were operated before the study and there was a lack of of adequate reliable records on coracohumeral and glenohumeral ligaments.

In this study, Constant-Murley, DASH and ASES, Turkish version of which were shown as valid and reliable before, were used as clinical evaluation methods (8-10, 15-19). Significant improvement was observed in all scores. Kim et al. (20) reported that ASES score was $90.8 \pm 6.5$ in patients with RI lesions in the postoperative $2^{\text {nd }}$ year. Lanz et al. (21) reported that postoperative Constant score was $81.2 \pm 14.6$ in patients who were repaired for anterior superior tears. The results of our study are similar to those studies (ASES: 95.82 \pm 7.82 , constant: 90.45 \pm 6.44 ) (ASES: 95.82 \pm 7.82 , constant: $90.45 \pm 6.44)$. DASH score was $5.99 \pm 13.68$ in our study which was also similar to those studies.

In this study, MRI was preferred as a radiological evaluation method. MRI is an accepted method for providing higher sensitivity compared to ultrasound, as well as not dependent on the individual and providing information about tendon quality beyond the tear. MRI is considered to be a reliable diagnostic examination in subscapularis tears (22). In our study, MRI in last control was used to see whether supraspinatus, infraspinatus and subscapularis tendons were intact. Radiological tendinopathy findings and tendon thicknesses were also recorded by using MRI. No patients with re-tear of supraspinatus or subchapularis tendons suggested that the surgical technique was effective. Only one patient had radiological tendinitis findings.

Small number of patients and no comparison made with other RC pathologies were the limitations of this study in which the clinical and radiological results of RI lesions were reported. RI lesions constitute a small portion of all RC tears which makes it difficult to report large series of cases. The inclusion of multiple clinical scores and the reported radiological results constituted the strengths of our study.

\section{Conclusion}

RI lesions constitute the least known group in all RC tears. Arthroscopic repair results show similar properties with other reported RC pathologies. Radiologically, the fact that there was no re-tearing suggests parallelism with clinical results. Comparative studies and studies on the natural course of RI lesions followed conservatively will enable a better understanding of this particular antithesis.

\section{Ethics}

Ethics Committee Approval: Koç University Faculty of Medicine Clinical Research Ethics Committee decision no: 2014.110.IRB1.001.

Informed Consent: It was not taken due to in vitro study.

Peer-review: Externally peer-reviewed.

\section{Authorship Contributions}

Concept: M.D., S.A., İ.E., Design: M.D., İ.E., H.Ö., Data Collection or Processing: İ.E., H.Ö., Ş.M.E., A.U., Analysis or Interpretation: İ.E., N.C., M.D., Literature Search: İ.E., H.Ö., Writing: İ.E., H.Ö., M.D.

Conflict of Interest: No conflict of interest was declared by the authors.

Financial Disclosure: The authors declared that this study received no financial support.

\section{References}

1. Warner JJ, Higgins L, Parsons IM 4th, Dowdy P. Diagnosis and treatment of anterosuperior rotator cuff tears. J Shoulder Elbow Surg 2001;10:37-46.

2. Collin P, Matsumura N, Ladermann A, Denard PJ, Walch G. Relationship between massive chronic rotator cuff tear pattern and loss of active shoulder range of motion. J Shoulder Elbow Surg 2014;23:1195-202.

3. Nho SJ, Frank RM, Reiff SN, Verma NN, Romeo AA. Arthroscopic repair of anterosuperior rotator cuff tears combined with open biceps tenodesis. Arthroscopy 2010;26:1667-74. 
4. Ide J, Tokiyoshi A, Hirose J, Mizuta H. Arthroscopic repair of traumatic combined rotator cuff tears involving the subscapularis tendon. J Bone Joint Surg Am 2007;89:2378-88.

5. Barth JR, Burkhart SS, De Beer JF. The bear-hug test: a new and sensitive test for diagnosing a subscapularis tear. Arthroscopy 2006;22:1076-84.

6. Gerber C, Hersche O, Farron A. Isolated rupture of the subscapularis tendon. J Bone Joint Surg Am 1996;78:1015-23.

7. Gerber C, Krushell RJ. Isolated rupture of the tendon of the subscapularis muscle. Clinical features in 16 cases. J Bone Joint Surg Br 1991;73:389-94.

8. Celik D. Turkish version of the modified Constant-Murley score and standardized test protocol: reliability and validity. Acta Orthop Traumatol Turc 2016;50:69-75.

9. Celik D, Atalar AC, Demirhan M, Dirican A. Translation, cultural adaptation, validity and reliability of the Turkish ASES questionnaire. Knee Surg Sports Traumatol Arthrosc 2013;21:2184-9.

10. Düger T, Yakut E, Öksüz Ç, Yörükan S, Bilgütay BS, Ayhan Ç. Reliability and validity of the Turkish version of the Disabilities of the Arm, Shoulder and Hand (DASH) Questionnaire. Turk J Physiother Rehabil 2006;17:99-107.

11. Jost B, Koch PP, Gerber C. Anatomy and functional aspects of the rotator interval. J Shoulder Elbow Surg 2000;9:336-41.

12. Lafosse L, Jost B, Reiland Y, Audebert S, Toussaint B, Gobezie R. Structural integrity and clinical outcomes after arthroscopic repair of isolated subscapularis tears. J Bone Joint Surg Am 2007;89:1 184-93.

13. Lafosse L, Lanz U, Saintmard B, Campens C. Arthroscopic repair of subscapularis tear: Surgical technique and results. Orthop Traumatol Surg Res 2010;96(8 Suppl):99-108.
14. Bennett WF. Subscapularis, medial, and lateral head coracohumeral ligament insertion anatomy: arthroscopic appearance and incidence of "hidden" rotator interval lesions. Arthroscopy 2001;17:173-80.

15. Constant CR, Gerber C, Emery RJ, Sojbjerg JO, Gohlke F, Boileau P. A review of the Constant score: modifications and guidelines for its use. J Shoulder Elbow Surg 2008;17:355-61.

16. Constant CR, Murley AH. A clinical method of functional assessment of the shoulder. Clin Orthop Relat Res 1987;214:160-4.

17. Beaton DE, Wright JG, Katz JN; Upper Extremity Collaborative Group. Development of the QuickDASH: comparison of three itemreduction approaches. J Bone Joint Surg Am 2005;87:1038-46.

18. Hudak PL, Amadio PC, Bombardier C. Development of an upper extremity outcome measure: the DASH (disabilities of the arm, shoulder and hand) [corrected]. The Upper Extremity Collaborative Group (UECG). Am J Ind Med 1996;29:602-8.

19. Richards RR, An KN, Bigliani LU, Friedman RJ, Gartsman GM, Gristina AG, et al. A standardized method for the assessment of shoulder function. J Shoulder Elbow Surg 1994;3:347-52.

20. Kim SJ, Jung M, Lee JH, Park JH, Chun YM. Arthroscopic repair of a significant ( $>50 \%)$ partial-thickness subscapularis tear concomitant with a full-thickness supraspinatus tear: technical considerations for subscapularis repair (transtendon technique versus tear completion). J Shoulder Elbow Surg 2015;24:875-81.

21. Lanz U, Fullick R, Bongiorno V, Saintmard B, Campens C, Lafosse L. Arthroscopic repair of large subscapularis tendon tears: 2- to 4-year clinical and radiographic outcomes. Arthroscopy 2013;29:1471-8.

22. Adams CR, Brady PC, Koo SS, Narbona P, Arrigoni P, Karnes GJ, et al. A systematic approach for diagnosing subscapularis tendon tears with preoperative magnetic resonance imaging scans. Arthroscopy 2012;28:1592-600. 